\title{
La producción laminar durante el Neolítico final y el Calcolítico: una renovación epistemológica
}

\author{
The blade production during the final Neolithic \\ and the Chalcolithic: an epistemological renewal
}

\author{
Juan Antonio Marín de Espinosa Sánchez \\ Sílex. Arqueología y Difusión del Patrimonio, S.L. \\ Calle Escuelas, 2, 2C E-30150 La Alberca, Murcia \\ info@tallarsilex.com
}

En este trabajo se analizan la evolución y las contribuciones realizadas al estudio de las producciones laminares de la Prehistoria reciente europea, con el objetivo de conocer las bases teórico-prácticas que han dado lugar al protocolo de investigación actual. Para ello, se han considerado desde estudios de diversos investigadores de finales del siglo XIx hasta la actualidad, prestando especial interés a los últimos cuarenta años de investigación arqueológica en Francia y en la península ibérica. Como resultado, tenemos la creación de una metodología en la que el referente arqueológico y la analogía experimental han aportado una nueva visión a los estudios tecnológicos.

\section{PALABRAS CLAVE}

TECNOLOGÍA LÍTICA, ARQUEOLOGÍA EXPERIMENTAL, NEOLÍTICO FINAL, CALCOLÍTICO

This paper analyses the evolution and contributions made to the study of blade productions in recent European prehistory, with the aim of knowing the theoretical and practical bases that have led to current research protocols.

To do this, I have considered from the studies of various researchers from the end of the $19^{\text {th }}$ century to the present. Special attention has been paid to the last forty years of archaeological research in France and the Iberian Peninsula. As a result, we have the creation of a methodology in which archaeological referents and experimental analogy has provided a new vision to technological studies.

\section{KeYWORDS}

LITHIC TECHNOLOGY, EXPERIMENTAL ARCHAEOLOGY, FINAL NEOLITHIC, CHALCOLITHIC 


\section{Introducción}

El conocimiento de la producción de útiles líticos tallados durante el Neolítico final y el Calcolítico (IV-III milenio aC), y en concreto de la producción laminar, es un elemento indispensable para la caracterización de los aspectos económicos en una sociedad (Binder et al., 1990; Pelegrin, 1991: 118; Manolakakis, 2005; Vaquer, 2007, 2012; Daura et al., 2015). En este sentido, los trabajos de investigación tecnológica suponen una apertura a fenómenos sociológicos asociados a nuevas formas de interacción entre grupos (Perlès, 2012: 539).

El congreso celebrado en 1964 en Les Eyzies de Tayac (Dordoña, Francia) supuso para los arqueólogos europeos la revelación, de la mano de Crabtree, de la técnica de presión para la obtención de productos laminares. Y será a partir de la década de los pasados años ochenta en la escuela francesa cuando comience una renovación epistemológica en el análisis de la talla laminar prehistórica (Tixier et al., 1980: 84; Inizan et al., 1995: 59, Pelegrin, 2005: 309) mediante el empleo del análisis tecnométrico y del método experimental (Binder y Pelegrin, 1983; Gallet, 1998; Pelegrin, 1988, 1997, 2002, 2006, 2012; Briois et al., 2005).

Por otra parte, el tratamiento de los datos tecnométricos y la caracterización de los estigmas en las series experimentales, a partir de análisis estadísticos, han permitido establecer índices y características de una técnica concreta, generando un referente experimental (Nami, 2011: 40).

A su vez, los análisis petrológicos de las materias primas han permitido la identificación de las litologías explotadas, lo que ha llevado a generar criterios de diferenciación que permiten identificar la fuente de materia prima explotada (Tarriño, 2001; Terradas et al., 2005; Mangado, 2006; Briois, 2006; Vaquer y Remicourt, 2009; Fernandes et al., 2013; Sánchez et al., 2014). De esta manera se está contribuyendo desde dos perspectivas a complementar la información de un útil prehistórico como elemento portador de una doble información, en lo espacial y en lo cultural (Mangado, 2006: 91), partiendo de la premisa de que un objeto de piedra es indisociable de su contexto geológico y arqueológico (Inizan et al., 1995: 91).

Además de estos avances a escala europea (Perlès, 1987, 1990, 2004; Mallet, 1992; Manolakakis, 1994; Millet-Richard, 1997; Riche, 1998; Ihuel, 2008; Guilbeau, 2010; Damlien, 2015), nuevos datos han permitido ampliar el conocimiento de las producciones laminares de la prehistoria reciente en Oriente Próximo (Altınbilek-Algül et al., 2012; Chabot y Pelegrin, 2012; Chabot, 2017; Pelegrin e Inizan, 2013) y en el continente americano (Gómez, 2011).

\section{Una historia de método y técnicas: el referente experimental}

La presencia o ausencia de estigmas tecnológicos en la industria lítica puede proporcionar información relevante en el diagnóstico de la técnica de talla empleada. En este sentido, 
el objetivo de la experimentación es establecer unos parámetros técnicos generales que ayuden al prehistoriador al reconocimiento de las técnicas de talla en el material arqueológico (Pelegrin, 1991: 122, 2006: 42).

Por otra parte, en las últimas décadas varios investigadores han desarrollado programas experimentales encaminados a la identificación de las técnicas de talla en la producción laminar del Neolítico final y del Calcolítico europeo, con el objetivo de conocer, identificar y sistematizar los diferentes parámetros técnicos (Binder y Pelegrin, 1983; Tixier, 1984; Pelegrin 1988, 1997, 1999, 2002, 2006, 2012; Briois et al., 2005; Inizan y Pelegrin, 2002: 106). De este modo, el análisis de las colecciones experimentales ha permitido trabajar con series completas que revelan para cada conjunto lítico tallado las condiciones precisas bajo las que se obtuvieron. Estos datos han facilitado el establecimiento de los criterios que caracterizan una técnica, así como el diagnóstico diferencial entre las distintas técnicas (Audouze et al., 2018).

\section{Los comienzos de la investigación del método laminar en Europa}

El reconocimiento de los métodos y las técnicas de la talla de útiles prehistóricos y el establecimiento de una correcta metodología para la interpretación de los restos arqueológicos, a partir de la práctica experimental, fueron esbozados por diversos investigadores a principios del siglo XIX, precursores del análisis empírico de las producciones líticas. Entre ellos encontramos al zoólogo y arqueólogo sueco Nilsson, que introdujo la etnografía como un nuevo enfoque de investigación en arqueología con su obra The primitive inhabitants of Scandinavia, traducida al inglés por Lubbock en 1868.

Por otra parte, la contribución de Evans al conocimiento de las producciones líticas talladas prehistóricas constituye un trabajo de referencia hasta la fecha. Un trabajo que fue publicado en su obra Ancient Stone Implements, weapons and ornaments of Great Britain (1872). En el capítulo XII, «Flint Flakes, cores», Evans establece analogías tecnológicas entre el material arqueológico y desarrolla experimentaciones encaminadas a reconocer el comportamiento técnico de los útiles del tallador, los estigmas producidos como consecuencia de la talla, así como la relación existente entre los ángulos de percusión y las materias primas empleadas. Evans se interesa también por las producciones de Grand Pressigny en su artículo «Palaeolithic Worked Flint Tools, Grand Pressigny» (1867).

En la escuela estadounidense será Holmes quien establezca una sistematización de las técnicas de talla, así como de los útiles del tallador empleados en la práctica experimental, a partir del uso de referencias etnográficas. Estos datos aparecen en su obra Handbook of aboriginal american antiquities (1919). Podemos atribuir a Holmes el inicio de los estudios modernos de la técnica de presión, así como de otros aspectos del análisis de los conjuntos líticos. 
En el aspecto tecnológico, Holmes distingue entre dos procesos de fractura: la percusión y la presión (Holmes, 1919: 283) y atribuye a Nilsson la identificación y la comprensión del empleo del percutor duro en piedra para la percusión directa, aportando nuevos aspectos como la variabilidad de tipos de percutores, en relación con la fase de trabajo (Holmes, 1919: 294, fig. 154) o el tiempo empleado en la ejecución de un útil (Holmes, 1919: 219). Su obra describe y muestra con fotografías y dibujos las técnicas de talla, los útiles del tallador empleados, los gestos técnicos y el resultado de su aplicación mecánica (Holmes, 1919: 301, figs. 164-166), resaltando la importancia del remontaje (Holmes, 1919: 293, fig. 153).

Por otra parte, será Ellis, en 1938, quien comience a llevar a cabo de una manera práctica una exploración de las técnicas descritas anteriormente por Holmes (Ellis, 1938, 1940). Sus trabajos aportan testimonios históricos y etnográficos sobre los procesos técnicos en la elaboración de puntas de proyectil ligeras y sobre la cadena operativa relacionada con los métodos, los útiles del tallador y las técnicas empleadas. La obtención de productos laminares en su mayor parte quedará relegada a unas cronologías antiguas. Esta información fue principalmente recopilada a través de los testimonios de personas que tuvieron contacto con grupos tribales del continente americano.

Es en ese momento cuando surge la confusión en relación con los útiles de talla empleados para la obtención de láminas por presión, ya que Ellis parte de la información recopilada por Sellers ${ }^{1}$ en 1886 y de los escritos de Torquemada ${ }^{2}$ para establecer los útiles del tallador empleados, en los que posteriormente Crabtree se fundamentará para establecer su hipótesis referente al proceso técnico empleado por los pueblos prehispánicos de México para la obtención de las láminas de obsidiana. Sin embargo, no será hasta 1981 cuando Clark reexamine el Códice Florentino, redescubra esta técnica a través del referente etnográfico y proceda a su identificación en el material arqueológico (Clark, 2012).

En Francia, a comienzos del siglo xx, y concretamente en 1907, Baudon, en su artículo "Le débitage et la taille du sílex», describirá los sistemas de producción de lascas y láminas y procederá a la caracterización de los soportes obtenidos con bulbos y conos de percusión, con el objetivo de adscribir la autoría de los útiles en piedra al ser humano. En 1928 De Givenchy, en su artículo "Le double cône de percussion", pondrá en relieve aspectos técnicos como el doble cono de percusión, documentado en materiales arqueológicos como los recuperados en Montmirail (Marne).

A su vez, el trabajo publicado en el Bulletin de la Société Préhistorique Française en 1931 por Cabrol y Coutier, titulado "Du conchoïde de percussion», analiza mediante analogía experimental los bulbos de percusión originados a partir del empleo de un percutor en madera, en piedra, y la identificación de los diversos estigmas que dejan la percusión directa con piedra y la percusión directa con madera.

1. Sellers se nutre de esta información para elaborar sus investigaciones publicadas en 1886 (Observation on stone chipping). Véase Sellers, 1886: 883, fig. 14, donde ya realiza un dibujo que adelanta el principio de aplicación de la fuerza y esquema de la palanca.

2. Hemos trabajado con la edición de 1723. 
Más tarde, la comunicación de Coutier, presentada en octubre de 1961 y publicada en 1962 bajo el título «Utilisation de la pression dans la taille de la pierre aux époques préhistoriques», pondrá en relieve la técnica de retoque mediante la aplicación de una presión con un presionador en asta de cérvido, hueso, piedra y bronce e intentará aplicar una lectura tecnológica a partir de los resultados de dicha experimentación.

Encontramos una perspectiva interesante y pionera sobre los estudios tecnométricos y el reconocimiento de estigmas en el material lítico tallado, en un artículo de Barnes y Cheyner, «Étude sur les techniques de débitage du silex et en particulier des nuclei prismatiques», publicado en 1935, en el que se establece un protocolo para el estudio de las producciones laminares a partir de la caracterización tecnológica de estigmas producidos como consecuencia del desprendimiento de una lasca o una lámina, que permiten reflexionar sobre el comportamiento técnico.

Por otra parte, a partir de la década de los años cincuenta, en el encuadre tipológico de la escuela de Burdeos se establecerá una caracterización tipológica de los conjuntos líticos que desembocará, a comienzos de los sesenta, en la tipología analítica de Laplace (1966).

A su vez, el concepto de cadena operativa establecido por Maget en 1953, en su artículo "Guide d'étude directe des comportements culturels», en el que se describen e individualizan los procesos técnicos que participan en la elaboración de un útil, desde la selección y adquisición de la materia prima hasta la transformación, uso, abandono y reciclaje, será empleado años más tarde por Leroi-Gourhan. Sus aportaciones se centran en la incorporación de la tecnología al marco de la investigación antropológica y su adaptación metodológica para el reconocimiento práctico de las etapas técnicas del proceso de producción del utillaje lítico tallado, ampliando de este modo la comprensión sobre la lectura de un útil y su estado técnico (Leroi-Gourhan, 1964, 1983).

En conclusión, esta primera etapa en el estudio de las producciones líticas talladas que engloba casi un siglo de investigaciones permitió establecer, a partir de pruebas experimentales, unas bases metodológicas para el estudio de las producciones líticas talladas, orientadas al reconocimiento de los diversos útiles de tallador empleados, los estigmas producidos por estos, así como los diversos estadios de la producción de un objeto. De este modo, la suma de estos conocimientos conformó una primera base de referencia en la disciplina arqueológica.

\section{Del congreso de Les Eyzies de Tayac al presente}

El congreso celebrado en Les Eyzies de Tayac entre los días 23 y 28 de noviembre de 1964 supuso la primera experiencia práctica conjunta en la que se expusieron los conocimientos técnicos adquiridos hasta la fecha por los arqueólogos franceses y sus colegas estadounidenses. La reunión, de la que no se conservan actas, se centró en los aspectos tecnológicos de los complejos líticos tallados con la aplicación práctica de métodos y técnicas de talla para la identificación de estigmas en los conjuntos arqueológicos (Texier y Meignen, 2011). 
Treinta y nueve años después de su celebración, Tixier, en el prefacio de la obra editada en 2003 por Hirth, Mesoamerican lithic technology: experimentation and interpretation, destaca la aportación al congreso de Les Eyzies de Tayac de los investigadores europeos que asistieron. Unas aportaciones que van en tres direcciones:

1. La introducción de la experimentación dentro del análisis de las producciones líticas talladas, aportando un nuevo método científico a sus análisis.

2. El inicio de la cooperación científica entre arqueólogos franceses y norteamericanos gracias a las lecciones impartidas por Crabtree a Bordes y Tixier.

3. La aplicación del tratamiento térmico en las producciones líticas talladas y el análisis de los efectos y las consecuencias que se observan en el material.

De entre sus participantes, las aportaciones más significativas vinieron de la mano de Bordes, con la exposición práctica de su experiencia personal en los estudios aplicados a las diferentes técnicas de talla del Paleolítico inferior y medio, así como el establecimiento de una tipología para la denominación de sus complejos industriales (Bordes, 1947, 1961a,b). Con ello Bordes sobrepasó a su antecesor Coutier, cuyas experiencias pueden sintetizarse en una primera aproximación de los comportamientos mecánicos de la talla, principalmente con percutores orgánicos (Coutier, 1929).

Por parte estadounidense, la aportación de Crabtree, que iniciaba sus investigaciones en la experimentación replicativa en la década de los años cincuenta, destacó, como hemos comentado anteriormente, por la revelación de la técnica de talla por presión de pie, a partir de estudios sobre la caracterización técnica de las producciones de láminas prismáticas elaboradas por los aztecas (Crabtree y Butler, 1964).

El conocimiento de la técnica de obtención de soportes laminares por presión (Crabtree, 1968) fue entonces asimilado por Tixier, quien identificó con posterioridad la técnica de presión en los desbastados estandarizados de Aïn Dokkara (Tébessa, Argelia), en la obtención de pequeñas láminas pertenecientes al Capsiense superior en el norte de África (Tixier, 1967, 1976; Tixier et al., 1980).

Fue entonces cuando Tixier trabajó en el reconocimiento y en la variabilidad técnica de los conjuntos arqueológicos del Capsiense superior y del Epipaleolítico del Magreb, con una amplitud cronológica que comprende desde el 9500 al 5500 BP (Rahmani, 2004: 89). A su vez, Tixier estableció los primeros criterios de reconocimiento de productos tallados por presión, publicados en 1976, destacando parámetros como la curvatura del perfil y la observación de los talones, con el objetivo de definir la materia prima de los punteros con los que se ejerce la presión.

Del mismo modo, propuso una nueva posición para la técnica de percusión indirecta, sous le pied (Tixier, 1972), dentro de un proceso de exploración de técnicas y adscribió el empleo de la misma a producciones laminares desde comienzos del Paleolítico superior. No obstante, en la actualidad el origen de la técnica de percusión indirecta se sitúa para en el Mesolítico final (7800 BP) en el Oeste de Europa (Pelegrin, 2006: 40). 


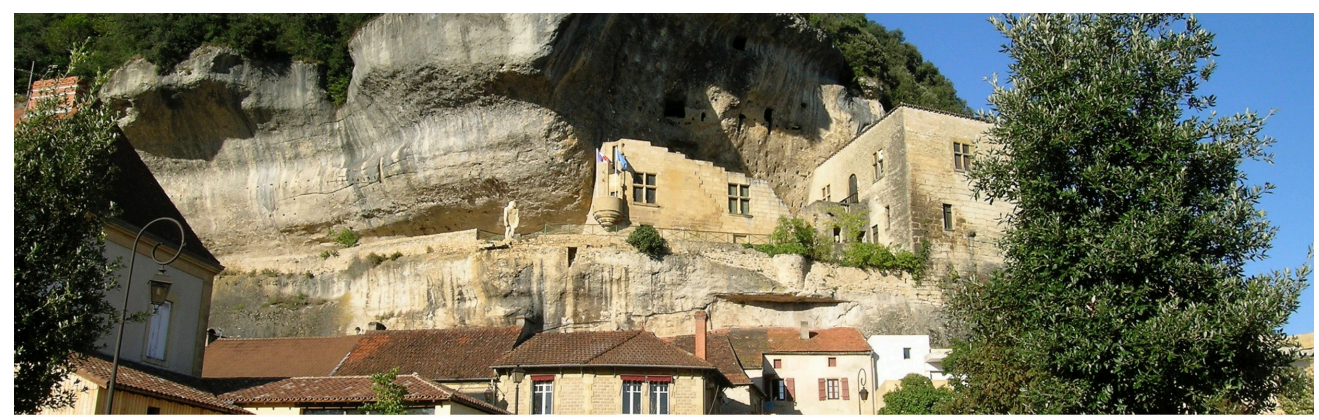

Figura 1. Museo Nacional de Prehistoria, Les Eyzies de Tayac.

Por lo tanto, la influencia de Crabtree en Tixier (Clark, 2012: 56) ha sido fundamental en cuestiones y enfoques experimentales relacionados con los estudios de la tecnología lítica, así como en el establecimiento y la estructuración de una terminología descriptiva en tecnología lítica (Tixier et al., 1980; Inizan et al., 1992, 1995, 1999). En este sentido, con el objetivo de definir la materia de los punteros con los que se ejerce la presión, en 1978 serán publicados diversos criterios de reconocimiento de productos tallados por presión, destacando parámetros como la curvatura del perfil y la observación de las características de los talones (Tixier, 1978).

Estas experiencias darán como resultado varias publicaciones (Inizan et al., 1976; Tixier et al., 1980), entre las que destaca la primera edición de Préhistoire de la pierre taillée (1980), cuyos autores, Tixier, Inizan y Roche, establecieron una sistematización en la lectura de los conjuntos líticos tallados desde una perspectiva tecnológica, aportando un léxico terminológico básico y descriptivo que ya fue publicado en 1963 por Tixier y traducido al inglés en 1974 por Newcomer.

A su vez, en España también fueron publicados diversos criterios para el análisis de los materiales líticos tallados en laboratorio (Merino, 1969; Bernaldo de Quirós et al., 1981).

Por otra parte, la variabilidad de las técnicas desarrolladas en la obtención de soportes laminares durante el Neolítico final y el Calcolítico revela el alto grado de conocimiento técnico alcanzado por determinados grupos, como consecuencia de un perfeccionamiento de la técnica y de la necesidad de abastecer la demanda de un tipo de producción determinada a unos grupos específicos.

En 1965 durante el simposio Background to evolution in Africa. Proceedings of a Symposium held at Burg Wartenstein, Austria, los conceptos de método y de técnica en tecnología lítica tallada fueron sistematizados por Tixier (Inizan, 2012: 14). En este sentido, el método comprende la relación en la organización de las extracciones de talla, el orden y la disposición en las tres dimensiones del espacio ${ }^{3}$ (Tixier, 1967: 807), identificado esencialmente por la lectura tecnológica individualizada de todos los restos líticos de un conjunto. De esta

3. Demostrativos, pero no necesarios, los esquemas diacríticos pueden objetivizar tales observaciones (Inizan et al., 1995: 131). 
manera se revela la secuencia de talla de la que provienen y se establece un «remontaje mental», capaz de enlazar y reordenar las secuencias observadas para reconstruir el método de talla ${ }^{4}$ (Inizan, 2002: 36), así como la valoración del conjunto que sustenta toda correcta práctica experimental (Pelegrin, 2006: 39).

Será a inicios de la década de los años ochenta, durante el ciclo de conferencias y los debates sobre tecnología lítica organizados en 1980 en Tervuren (Bélgica) y en 1982 en Meudon (Francia), cuando se establezca un protocolo para el estudio de las colecciones líticas talladas, relegando los estudios tipológicos tradicionales (Inizan, 2012: 15).

Hay que destacar que, durante las últimas cuatro décadas, el reconocimiento de las técnicas de talla ha sufrido un avance epistemológico debido a la aportación del referente experimental al análisis de las producciones laminares (Tixier, 1972; Newcomer, 1975; Olausson, 1983; Pelegrin, 1984a, 1984b; Texier, 1984). Si bien es cierto que el método experimental debe ir asociado a una base teórica, este tiene su génesis en la práctica experimental (Nami, 2010), estableciendo una madurez en la resolución exitosa de problemas y conformando a su vez una tradición científica (Kuhn, 1970: 169), convirtiéndose en una auténtica disciplina (Melogno, 2012: 22).

En este sentido, el simposio celebrado en Meudon-Bellevue (Francia), durante los días 27 a 29 de octubre de 1982 organizado por el CNRS, que será publicado dos años más tarde (Tixier, 1984) bajo el título Préhistoire de la pierre taillée 2. Économie du débitage laminaire (CREP 1984), supuso una recopilación de conocimientos sobre la caracterización tecnológica de las producciones laminares desde una perspectiva experimental. Sus aportaciones pueden sintetizarse en cuatro apartados:

1. Ofrece un estado de la cuestión sobre el conocimiento de las producciones laminares, principalmente obtenidas mediante la técnica de presión, a partir de referentes etnohistóricos y arqueológicos.

2. La técnica de presión para la obtención de soportes laminares, la variabilidad morfológica de núcleos, así como su desarrollo volumétrico durante el proceso de talla y la relación existente con los efectivos laminares.

3. Los sistemas de inmovilización de los núcleos para el desbastado por presión y su relación con la ausencia de ondulaciones marcadas en la cara inferior de los efectivos laminares.

4. La propagación de las fracturas por presión sobre sólidos homogéneos e isótropos y la mejora del sílex a partir del tratamiento térmico.

Será en 1992 cuando aparezca una nueva edición en lengua inglesa, Technology of Knapped Stone (Inizan et al., 1992), en la que se exponen los últimos conocimientos de la

4. Sin embargo, la observación aislada de piezas talladas no permite reconocer directamente las técnicas, ya que parámetros como el modo de aplicación de la fuerza, el aspecto de los útiles y los gestos pueden inducir a errores en la interpretación del diagnóstico de la técnicas de talla (Pelegrin, 2000: 74). 

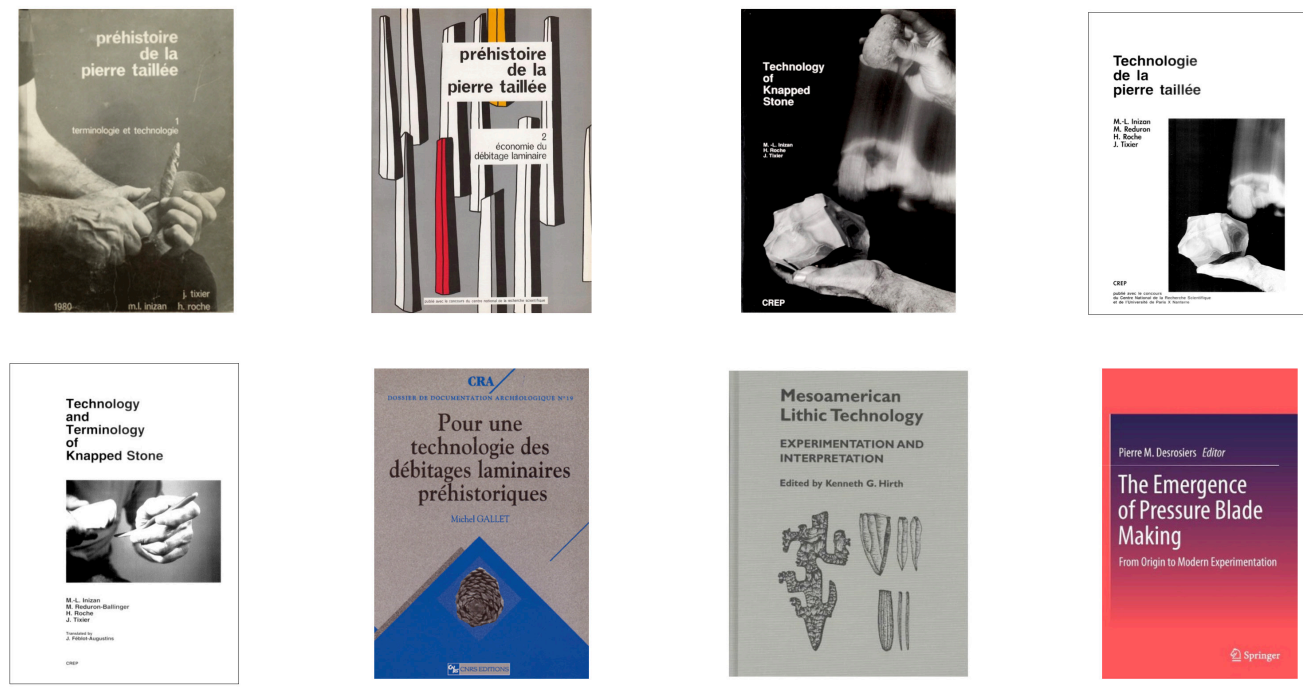

Figura 2. Portada de varias obras expuestas en el texto.

tecnología, tanto en el campo teórico como en sus aplicaciones prácticas, con un vocabulario multilingüe. ${ }^{5}$ En esta obra se presenta un criterio uniforme en el lenguaje descriptivo, adoptando términos y expresiones, en un primer intento de normalización y clarificación del registro material tallado. Además, se establecen conceptos fundamentales como la aptitud y caracterización de las materias primas susceptibles de la talla, la economía de los desbastados, los útiles de talla y los distintos métodos y técnicas identificados a lo largo de la Prehistoria. Se incorpora también la exposición de criterios para la lectura tecnológica, con una ilustración gráfica y fotográfica destinada a la comprensión de los diferentes fenómenos explicados. En una segunda parte, se unifica un léxico terminológico especializado que fue ampliado en 1995 en el capítulo 8.6

Por otra parte, la edición de 1995 fue realizada a partir de la edición inglesa de 1992, con un capítulo dedicado a la representación gráfica de los útiles en piedra tallada y firmado por Reduron-Ballinger (Inizan et al., 1995). En el capítulo séptimo, expone los principios generales y los criterios convencionales para la representación gráfica de un objeto lítico tallado. En 1999, aparece el tomo 5 de la colección que deriva de la edición francesa de 1995, traducida al inglés por Féblot-Augustins con el título de Technology and Terminology of Knapped Stone (Inizan et al., 1999).

En 1998 se publica la obra de Gallet Pour une technologie des débitages laminaires préhistoriques, en la que se exponen los resultados de su investigación (Gallet y Texier, 1988, 1991a,b), que tiene por objeto el conocimiento de las producciones laminares prehistóricas

5. Inglés, árabe, francés, alemán, griego, italiano, ruso, castellano.

6. Inglés, árabe, francés, alemán, griego, italiano, castellano y portugués. 
mediante el análisis tecnométrico y el reconocimiento de estigmas de series experimentales, en un intento de establecer una relación entre las características tecnológicas de los productos laminares y las técnicas de talla empleadas.

El material objeto de estudio consistió en 30 series experimentales elaboradas por cuatro investigadores con distintos grados de madurez y conocimientos técnicos: Texier, Pelegrin, Bodu y tres series complementarias de Tixier que fueron empleadas a comienzos de este estudio en 1981. Las técnicas analizadas fueron la percusión directa, la percusión indirecta y la presión, con un total de 1.493 láminas estudiadas. Cada efectivo laminar fue objeto de un análisis que combinó los parámetros métricos y los cualitativos.

Este trabajo ha de considerarse como una obra abierta y, por tanto, debe ser ampliado y contrastado con nuevas series experimentales que permitan establecer relaciones entre las condiciones y las características de los productos obtenidos.

Por otra parte, el congreso organizado entre el 22 y el 28 de mayo de 2000 por el Departamento de Antropología de la Penn State University (Pensilvania, Estados Unidos), estableció una nueva perspectiva metodológica en el análisis de las producciones laminares en obsidiana mediante la técnica de presión. Los resultados de este encuentro fueron editados por Hirth en 2003 y dedicados a Crabtree con el título Mesoamerican Lithic Technology: Experimentation and Interpretation. Estos resultados representan una vía de apertura al conocimiento de la tecnología lítica tallada de Mesoamérica, cuya aportación puede encuadrarse en tres grandes apartados:

1. En el primero de ellos se evalúa y plasma el estado del conocimiento de la producción de láminas prismáticas en obsidiana en Mesoamérica (Hirth, 2003: 6).

2. En un segundo apartado se aplican programas experimentales destinados a establecer medidas relativas a la morfología global de los efectivos laminares, así como a la posible intervención de diversas técnicas dentro de la cadena operativa para la obtención de láminas prismáticas por presión. En especial, se menciona la percusión indirecta como técnica empleada para las preparaciones o para la obtención de las láminas.

3. Por último, se establecen modelos de análisis experimentales que pueden ser introducidos en el estudio y la interpretación de las colecciones arqueológicas.

Encontramos la última gran aportación al estudio de las producciones laminares en la Prehistoria reciente en la obra editada por Desrosiers, en 2012, The Emergence of Pressure Blade Making, from Origin to Modern Experimentation. Esta obra ofrece un compendio de trabajos recientes, así como un mapa de distribución de la caracterización técnica de la talla por presión en el tiempo y en el espacio. La obra queda dividida en tres grandes apartados: el primero dedicado a la historia de la investigación; el segundo, a la producción de láminas por presión a escala mundial, y el tercero, a los recientes avances en la experimentación. Este trabajo constituye una obra de referencia que plasma el conocimiento global que caracteriza las producciones laminares por presión a escala mundial, sistematizando tecnológicamente los modos de presión y sus características (Pelegrin, 2012). 

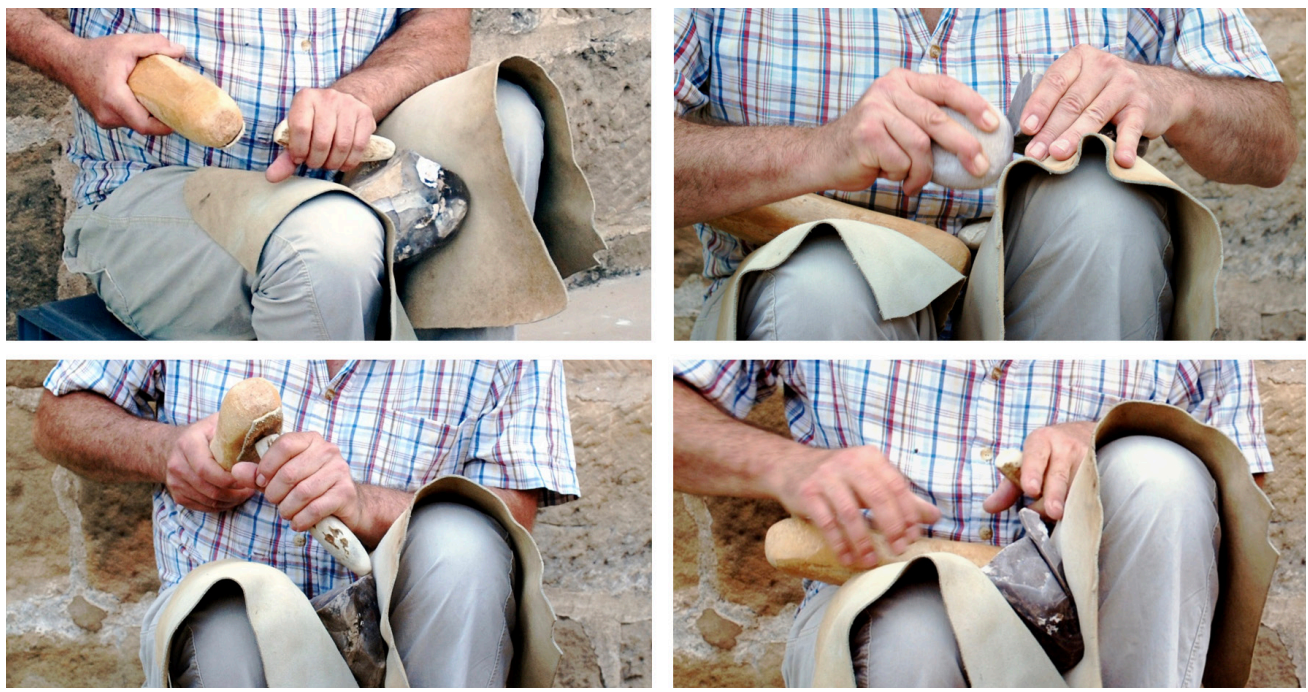

Figura 3. Proceso de obtención de una lámina mediante percusión indirecta. Demostración comentada realizada por Jacques Pelegrin, Caspe, 2012.

Por tanto, desde el congreso de Les Eyzies de Tayac de 1964 hasta la actualidad, el conocimiento de las producciones laminares durante el Neolítico final y el Calcolítico ha sufrido un avance exponencial, estableciendo en la disciplina unos criterios descriptivos a partir de la exploración sistemática de las técnicas de talla, que ha proporcionado un referente experimental que ha permitido establecer el papel que desempeña cada elemento dentro de la cadena operativa.

De este modo, la consulta de las colecciones experimentales se ha convertido en una herramienta fundamental para el establecimiento de hipótesis empíricas, referenciadas y bien documentadas de la técnica o la variabilidad de técnicas presentes en un conjunto arqueológico. De esta manera se convierte el referente experimental adaptado a cada serie arqueológica en un elemento indisociable en el estudio del material arqueológico, que permite identificar las particularidades concretas de una técnica, así como la interacción entre grupos y el alcance de su difusión.

\section{Los estudios tecnométricos y experimentales en la península ibérica}

A partir del VI International Flint Symposium celebrado en Madrid en 1991 y la publicación en 1997 por la Universidad de Granada del volumen Siliceous Rocks and Culture, en el que aparece el artículo escrito por Martínez Late Prehistory Blade production in Andalusia (Spain), el interés 
en las dos últimas décadas por las producciones laminares durante la Prehistoria reciente en la península ibérica ha pasado, de un enfoque descriptivo y tipológico, a la incorporación de la analogía experimental, la contextualización petrológica y los estudios funcionales, revelando la caracterización de ciertas producciones y las interacciones que se establecen entre grupos humanos (Gibaja et al., 2004, 2009; Vaquer y Briois, 2006; Pelegrin y Morgado, 2007; Vaquer y Remicourt, 2009; Vaquer, 2012; Morgado y Martínez, 2013; Daura et al., 2015).

Este cambio epistemológico en la interpretación de las producciones laminares a partir del referente experimental en la Prehistoria reciente de la península ibérica ha comenzado a generarse en los últimos años como consecuencia de tres factores interrelacionados:

1. El estudio de Pelegrin y Morgado «Primeras experimentaciones sobre la producción laminar del Neolítico reciente-Edad del Cobre del sur de la península ibérica», presentado en el primer Congreso Español de Arqueología Experimental, celebrado en Santander del 24 al 25 de noviembre de 2005 por la Asociación Española de Arqueología experimental Experimenta y publicado en 2007, comprende la preparación del núcleo, el procedimiento para la extracción de las láminas y el establecimiento de unos elementos de referencia para el reconocimiento de la técnica de talla mediante presión reforzada con palanca y el uso de puntero de cobre. El programa experimental desarrollado por Pelegrin y Morgado estaba compuesto de tres series que suman un total de 68 láminas. De ellas, 13 grandes láminas fueron extraídas con palanca y dos series empleando la presión de pie con muleta, en la que intervinieron un puntero en cobre y otro en asta de cérvido. A su vez, se empleó un punch con un puntero en cobre para la confección de las aristas durante la elaboración del núcleo para la obtención de grandes láminas.

2. Por otra parte, del 24 al 29 de septiembre de 2007, se desarrolló en el Centro de Educación Algaba de Ronda (Málaga) el curso «Tecnología lítica tallada prehistórica y experimentación», bajo la Dirección de Martínez y Morgado, a través del Centro Mediterráneo de la Universidad de Granada. En él colaboraron el Departamento de Prehistoria y Arqueología de la Universidad de Granada, el Centro Algaba de Ronda (Málaga) y el Taller de Arqueología Experimental de Ronda (T.A.E.R.). Este curso se desarrolló dentro del proyecto de investigación Producción lítica especializada durante la Prehistoria reciente de la península Ibérica (HUM2006-13635). Los responsables científicos del curso fueron J. Pelegrin y P-J. Texier, que impartieron el curso junto a S. Maury.

Esta reunión facilitó la transmisión de conocimientos técnicos y aspectos metodológicos por parte de los docentes franceses, impulsando los trabajos y la revisión de las colecciones arqueológicas desde dos visiones:

1. La relevancia que ha representado el reconocimiento de las técnicas de talla para los estudios en tecnología lítica y experimentación desde el congreso de Les Eyzies de Tayac hasta la actualidad. 
2. El reconocimiento de los distintos métodos, técnicas y operaciones de talla documentados en las producciones líticas desde el Paleolítico inferior hasta la Prehistoria reciente, principalmente en el ámbito europeo.

En este apartado es necesario hacer una recapitulación de las aportaciones de Pelegrin asociadas al reconocimiento de las técnicas de talla en las producciones laminares de la Prehistoria reciente (Pelegrin 1984a,b,c, 1988, 1991, 2002, 2006, 2012; Pelegrin et al., 1999, $2000,2002,2006)$. Sus aportaciones han sido obtenidas a partir de la evaluación de series experimentales por percusión indirecta, ${ }^{7}$ deducidas de la observación de los núcleos denominados «libras de mantequilla» (Mallet et al., 1994; Pelegrin e Ihuel, 2005). Sus observaciones establecen modelos tecnoeconómicos durante el Neolítico final en Grand Pressigny, en la extracción de la materia prima y en la reconstrucción de los gestos técnicos (Pelegrin, 2002). Pelegrin establece sistemas de reducción según su orden de desbastado y la clasificación del tipo de láminas, y proporciona una valoración económica de la producción (Pelegrin, 1997).

A su vez, Pelegrin ha establecido los parámetros técnicos de presión con palanca a partir de experimentaciones desarrolladas en 1983 y durante 1990-1995 en el Archéodrome de Beaune (Francia), con un único parámetro técnico en la morfología de la pieza intermedia que transmite la presión (puntero de madera, asta de ciervo o cobre), con un total de cincuenta series talladas mediante presión con palanca, estableciendo unos criterios distintivos entre la percusión indirecta y la técnica de presión con palanca (Pelegrin y Morgado, 2007: 135).

Por otra parte, la distinción de parámetros como el principio de aplicación de la fuerza (percusión directa o indirecta, presión), la naturaleza y morfología de los útiles de talla, o el modo de sujeción de los núcleos (Pelegrin, 1984a, 1984c, 1988, 2002, 2003, 2006, 2012) conduce el análisis tecnológico a aspectos cuantitativos y cualitativos de la producción, derivando en implicaciones de la conducta humana y la valorización social de la producción.

Por último, el desarrollo de la exposición itinerante Europa al final de la Prehistoria. Las grandes hojas de sílex generó en Barcelona el workshop ${ }^{8}$ internacional La península Ibèrica al final de la Prehistòria: les grans làmines de sílex, celebrado los días 9 y 10 de junio de 2008 en el Museu d'Arqueologia de Catalunya (Barcelona).

La recapitulación en las últimas décadas de los conocimientos sobre producciones laminares dentro del ámbito peninsular se puso de manifiesto en la publicación de las actas de este congreso por dicha institución en 2009, bajo el título Les grans làmines de sílex. Europa al final de la Prehistòria. Este trabajo es un referente básico en el estudio de las producciones laminares de la península ibérica y del sur de Francia, en la que destacan las siguientes aportaciones:

- El conocimiento de las producciones laminares tanto en el Mediodía francés (Vaquer y Remicourt, 2009) como en el ámbito peninsular.

7. Lejre, Dinamarca, 1986-1989.

8. Esta exposición está asociada a la reunión internacional dirigida por Marquet y Verjux en Tours (Indre-et-Loire, Francia), el 7 de septiembre de 2007. 


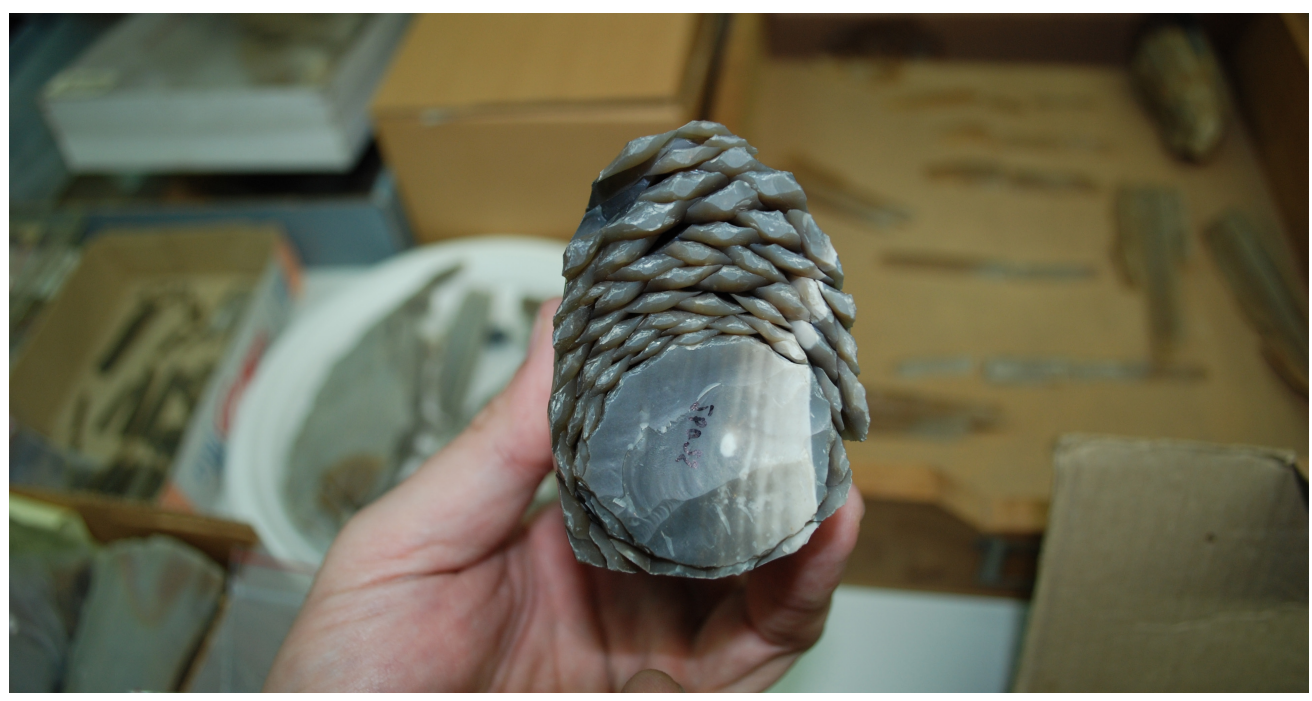

Figura 4. Remontaje experimental de un núcleo laminar. Experimentación realizada por Jacques Pelegrin.

- Redes de intercambio, áreas de producciones especializadas y talleres de producción doméstica que se documentan en el área peninsular.

- Queda establecido como gran lámina9 aquella con dimensiones longitudinales mayores de $200 \mathrm{~mm}$, mientras que la anchura podría estar en el límite de los $22 \mathrm{~mm}$, para las láminas talladas por presión. Este último umbral marca el inicio a partir del cual debió utilizarse el sistema de presión con palanca (Pelegrin, 2002, 2006).

De este modo, los conocimientos en la disciplina obtenidos de las producciones laminares de la Prehistoria reciente europea han sido canalizados hacia el estudio tecnológico de las producciones especializadas en la península ibérica, revelando, junto a la identificación petrológica, los talleres de producción laminar especializada que han permitido establecer la adscripción de distintas tradiciones culturales y la interacción que se establece entre grupos.

\section{Consideraciones finales}

El proceso de redescubrimiento de los conocimientos técnicos desaparecidos de la memoria colectiva ha partido del análisis de las colecciones arqueológicas y de la práctica experimen-

9. La definición de una gran lámina de sílex viene establecida desde hace tiempo (Siret, 1892: 391-392) y por trabajos posteriores (Tixier, 1984; Pelegrin, 1984, 1988, 2002, 2003, 2006; Inizan y Lechevallier, 1995; Martínez y Morgado, 2005; Pelegrin y Morgado, 2007, 2008). 
tal. Esta renovación epistemológica que se originó en Europa a partir del congreso de Les Eyzies de Tayac en 1964 fue consolidándose durante la década de los años setenta (Tixier, 1972) y sistematizándose a partir de la década siguiente (Tixier et al., 1980; Tixier, 1984; Texier, 1982, 1984; Binder y Pelegrin, 1983; Pelegrin 1984a,b,c, 1988a, 1991, 2002, 2006, 2012, Pelegrin y Morgado, 2007). De este modo, el método consensuado para el análisis de las producciones laminares puede desarrollarse a partir de dos variantes.

La primera de ellas debe su génesis a la aplicación de un programa experimental para la resolución de cuestiones en las que se hace necesario el desarrollo de la práctica experimental, que permite establecer referentes técnicos, aspectos cuantitativos y cualitativos de una producción determinada y que han dado pie a referentes experimentales.

Encontramos una segunda variante en aquellos estudios que plantean hipótesis tecnológicas a partir de referentes experimentales y que han permitido interpretar y relacionar las principales características técnicas en la producción laminar de la Prehistoria reciente (Inizan, 2002; Léa 2004; Manolakakis, 2005; Ihuel, 2008; Remicourt et al., 2009; Guilbeau, 2010; Gómez, 2011, 2012; Marín de Espinosa et al., 2011, 2013; Léa et al., 2012).

\section{Agradecimientos}

El presente trabajo se enmarca en la tesis doctoral del autor. Quiero agradecer a mis codirectores de tesis doctoral, el Dr. Jean Vaquer (TRACES, CNRS), el Dr. Xavier Mangado Llach (Universitat de Barcelona) y el Dr. Ignacio Martín-Lerma (Universidad de Murcia), su vinculación y generosa dedicación a este proyecto.

También quiero agradecer al Dr. Jacques Pelegrin (PréTech, CNRS) las apreciaciones hechas en mi tesis doctoral durante mi estancia en su laboratorio de Nanterre.

\section{Bibliografía}

ALTINBILEK-ALGÜL, C., ASTRUC, L., BINDER, D., PELEGRIN, P., 2012, Pressure blade production with a lever in the Early and Late Neolithic of the Near East, en P.M. DESROSIERS (ed.), The Emergence of Pressure Blade Making, Springer, Nueva York, 157-179.

AUDOUZE, F., BODU, P., KARLIN, C., JULIEN, M., PELEGRIN J., PERLÈS C., 2018, Leroi-Gourhan and the chaîne opératoire: a response to Delage, World Archaeology, 49-5, 718-723.
BARNES, A.S., CHEYNIER, A., 1935, Étude sur les techniques de débitage du silex et en particulier des nuclei prismatiques, Bulletin de la Société Préhistorique Française 32, n. ${ }^{\circ 5}$, 288-299.

BAUDON, T., 1907, Le débitage et la taille du silex, Bulletin de la Société Préhistorique Française 4, n. ${ }^{\circ} 8,423-426$.

BERNALDO DE QUIRÓS F., CABRERA V., CACHO C., VEGA L.G., 1981, Proyecto de análisis técnico para las industrias líticas, Trabajos de Prehistoria 38, 9-37. 
BINDER D., PELEGRIN J., 1983, Expérimentation sur la technologie lithique du Chasséen, Campagne expérimentale 1983, Beaune, Archéodrome.

BINDER, D., PERLÈS, C., INIZAN, M. L., LECHEVALLIER, M., 1990, Stratégies de gestion des outillages lithiques au Néolithique, Paleo 2, 257-283.

BORDES, F., 1947, Étude comparative des différentes techniques de taille du silex et des roches dures, L'Anthropologie 51, 1-29.

BORDES, F., 1961a, Typologie du Paléolithique ancien et moyen, tome 1, Bordeaux, Publications de l'Institut de Préhistoire de l'Université de Bordeaux, Imprimerie Delmas, Mémoire n. ${ }^{\circ} 1$.

BORDES, F., 1961b, Typologie du Paléolithique ancien et moyen, tome 2 (planches), Bordeaux, Publications de l'Institut de Préhistoire de l'Université de Bordeaux, Imprimerie Delmas, Mémoire $\mathrm{n} .{ }^{\circ} 1$.

BRIOIS, F., 2005, Les Industries de pierre taillée néolithiques en Languedoc occidental : nature et évolution des outillages entre les VI et III millénaires av. J.-C., Lattes, Association pour le développement de l'archéologie en LanguedocRoussillon, Monographies d'Archéologie Méditerranéenne, 341.

BRIOIS, F. 2006, Un atelier de production laminaire chalcolithique dans la région des minières de silex de Collorgues (Gard), en J. VAQUER, y F. BRIOIS (dir.), La fin de l'Âge de Pierre en Europe du Sud : matériaux et productions lithiques taillées remarquables dans le Néolithique et le Chalcolithique du Sud de l'Europe, Actes de la table ronde de l'EHESS, Carcassonne, 5-6 sept. 2003, Tolosa, éd. AEP, 165-174.

BRIOIS, F., NEGRINO, F., PELEGRIN, J., STARNINI, E., 2005, Flint exploitation and blade production during the Harappan period (Bronze Age): testing the evidence from the Rohri Hills flint mines (Sindh-Pakistan) throughout an experimental approach, en Stone Age-Mining Age, Proceedings of the VIII International Flint Symposium, September 13-17, 1999, Bochum, (Körlin, G., \& Weisgerber, G., Eds.), Veröffentlichungen aus dem Deutschen Bergbaumuseum Bochum
148, Der Anschnitt Beiheft 19, Deutschen Bergbaumuseum, Bochum,185-191.

CABROL, A., COUTIER, L., 1931, Du conchoïde de percussion, Bulletin de la Société Préhistorique Française 28, n. ${ }^{\circ}$ 6, 321-322.

CHABOT, J., 2017, Industrie néolithique de longues lames en obsidienne, l'exemple d'Aknashen-Khatunarkh (Arménie, début du VI ${ }^{\mathrm{e}}$ millénaire) : sur la piste des premiers débitages par pression, Journal of Lithic Studies 4-2 (en línea). Consulta: 5 de junio de 2018. Disponible en http://journals.ed.ac.uk/lithicstudies/.

CHABOT, J., PELEGRIN, J., 2012, Two Examples of Pressure Blade Production with a Lever: Recent Research from Southern Caucasus (Armenia) and Northern Mesopotamia (Syria, Iraq), en P. M. DESROSIERS (ed.), The Emergence of Pressure Blade Making, Springer, Nueva York, 181-198.

CLARK, J. E., 2012, Stoneworkers' approaches to replicating prismatic blades, en P. M. DESROSIERS., (ed.) The Emergence of Pressure Blade Making, Springer, Nueva York, 43-105.

COUTIER, L., 1929, Expériences de taille pour rechercher les anciennes techniques paléolithiques, Bulletin de la Société Préhistorique Française 26, 172-174.

COUTIER, L., 1962, Utilisation de la pression dans la taille de la Pierre aux époques préhistoriques, Bulletin de la Société Préhistorique Française 59, n. ${ }^{\circ}$ 5-6, 354-356.

CRABTREE, D.E., 1968, Mesoamerican polyhedral cores and prismatic blades, American Antiquity 33 (4), 446-478.

CRABTREE, D.E., BUTLER B.R., 1964, Notes on experiments in flintknapping: 1, Heat treatment of silica materials, Tebiwa 7 (1), 1-6,

DAMLIEN, H., 2015. Striking a Difference? The effect of knapping techniques on blade attributes. Journal of Archaeological Science 63, 122-135.

DAURA, J., SANZ, M., OMS, F. X., PEDRO, M., MARTÍNEZ, P., RUBIO, A., TEJERO J.-M., MANGADO, X., VAQUER, J., LÓPEZ-CACHERO, J., OLIVA, M., ASENSIO, A., ÁlVAREZ, R., 
FULLOLA, J.M., PETIT, M.A., 2015, La Cova de l'Avi (Vallirana, Barcelona) y el inicio del Neolítico final en el Nordeste de la Península Ibérica. Inhumaciones colectivas y nuevas redes de intercambio, Trabajos de Prehistoria 72-2, 327-341.

DE GIVENCHY, P., 1928, Le double Cône de Percussion (Conoïdes), Bulletin de la Société Préhistorique Française 25, n. ${ }^{\circ 5}$, 253-255.

DESROSIERS P. M., 2012, The Emergence of Pressure Blade Making, Springer, Nueva York.

ELLIS, H. H., 1938, Lithic Problems, American Antiquity 4-1,63-64.

ELLIS, H.H., 1940, Flint-working Techniques of the American Indians: an experimental study, Ohio Sate Museum, Columbus.

EVANS, J., 1868, Palaeolithic Worked Flint Tools, Grand Pressigny, Journal Offrint, Londres.

EVANS, J., 1872, The Ancient Stone Implements, Weapons and Ornaments of Great Britain, Londres, 2. ${ }^{\text {a }}$ ed. 1897.

FERNANDES, P., RAYNAL, J.-P., TALLET, P., TUFFERY, C., PIBOULE, M., SÉRONIEVIVIEN, M., SÉRONIE-VIVIEN, M.-R., TURQ, A., MORALA, A., AFFOLTER, J., MILLET, D., MILLET, F., BAZILE, F., SCHMIDT, P., FOUCHER P., DELVIGNE V., LIAGRE, J., GAILLOT, S., MORIN, A., MONCEL, M-H., GARNIER J. F., LÉANDRI-BRESSY, C., 2013, Une carte et une base de données pour les formations à silex du sud de la France: un outil pour la pétroarchéologie, Paléo: revue d'archéologie préhistorique 24, 219-228.

GALLET, M., 1998, Pour une technologie des débitages laminaires préhistoriques, Dossier de documentation archéologique 19, CNRS, París, reedición 2002.

GALLET, M., TEXIER, P. J., 1991a, Un appareil de mésures pour caracterisation et révaluation des contraintes exercées sur un nucleus lors d'un débitage expérimental des lames par pression, Archéologie expérimental, tome 2, La Terre : l'os et la pierre, la maison et les champs, Actes du Colloque International Expérimentation en archéologie: Bilan et perspectives, Archéodrome de Beaune,
6-9 avril, 1988, Collection Ar, París, Éditions Errance, 102-109.

GALLET, M., TEXIER, P. J., 1991b, Caracterisation et evaluation des contraintes lors d'un débitage experimental de lames par pression, perspectives experimentales et implications archéologiques, La pierre préhistorique, París, 127-132.

GIBAJA, J. F., PALOMO, A., TERRADAS, X., CLOP, X., 2004, Útiles de siega en contextos funerarios del 3500-1500 cal ANE en el noreste de la Península ibérica: El caso de las grandes hojas de sílex, Cypsela 15, 187-195.

GÓMEZ, Y. A., 2011, Industries lithiques à composante lamellaire par pression du Nord Pacifique de la fin du Pléistocène au début de l'Holocène : de la diffusion d'une technique en Extrême-Orient au peuplement initial du Nouveau Monde, Laboratoire Préhistoire et Technologie, Maison de l'Archéologie et de l'Ethnologie, École doctorale Milieux, Cultures et Sociétés du Passé et du Présent,

GÓMEZ, Y.A., 2012, Pressure Microblade Industries in Pleistocene-Holocene Interior Alaska: Current Data and Discussions, en P. M. DESROSIERS (ed.), The Emergence of Pressure Blade Making, Springer, Nueva York, 347-374.

GUILBEAU, D., 2010, Les grandes lames et les lames par pression au levier du Néolithique et de l'Énéolithique en Italie, Université Paris OuestNanterre, La Défense (tesis de doctorado).

HIRTH, K., 2003, Mesoamerican Lithic Technology: Experimentation and Interpretation, The University of Utah Press, Salt Lake City.

HOLMES, W. H., 1919, Handbook of Aboriginal American Antiquities, Part I: Introductory, the Lithic Industries, The Smithsonians Institution Bureau of American Ethnology 60, Washington D.C.

IHUEL, E., 2008, De la circulation des lames à la circulation des poignards. Mutations des productions lithiques spécialisées dans l'Ouest de la France du Ve au III millénaire, Université de Paris X Nanterre, 2 vol. (tesis de doctorado).

INIZAN, M.-L., 2002, Tailler des roches par pression: émergence d'une technique, étapes de sa diffusion dans le monde, en J. GUILAINE (ed.), 
Matériaux, productions, circulations du Néolithique à l'Âge du Bronze, Éditions Errance, París, 33-46.

INIZAN, M.-L., 2012, Pressure débitage in the Old World: Forerunners, researches, geopoliticshanding on the Baton, en P.M. DESROSIERS (ed.), The Emergence of Pressure Blade Making, Springer, Nueva York, 11-42.

INIZAN, M.-L., ROCHE, H., TIXIER, J., 1976, Avantage d'un traitement thermique pour la taille des roches siliceuses, Quaternaria, Roma XIX, 1-18.

INIZAN, M.-L., ROCHE, H., TIXIER, J., 1992, Technology of Knapped Stone. Préhistorie de la Pierre Taillée, 3, Cercle de Recherches et d'Études Préhistoriques, Meudon.

INIZAN, M.-L., REDURON, M., ROCHE, H., TIXIER, J., 1995. Technologie de la Pierre Taillée, 4, Cercle de Recherches et d'Études Préhistoriques, Meudon.

INIZAN M. L., LECHEVALIER, M., 1995, A Transcultural Phenomenon in the Chalcolithic and Bronze Age lithics of the Old World: Raw Material Circulation and production of Standardized Long Blades. The Example of the Indus Civilization, South Asian Archaeology, 77-85.

INIZAN, M.-L., REDURON, M., ROCHE, H., TIXIER, J., 1999, Technology and Terminology of Knapped Stone, Cercle de Recherches et d'Études Préhistoriques, París.

INIZAN, M.-L., PELEGRIN, J., 2002, Débitage par pression et expérimentation : une question de méthodologie, Paléorient 28 (2), 105-108.

KUHN, T., 1970, Consideraciones en torno a mis críticos, en El camino desde la Estructura, Barcelona, Paidós, 2002, 151-209.

LAPLACE, G., 1966, Pourquoi une typologie analytique?, L'Anthropologie 70, 1-2, 193-201.

LÉA, V., 2004, Les industries lithiques du Chasséen en Languedoc oriental : caractérisation par l'analyse technologique, British Archaelogical, Reports International Series 1232, Oxford.

LÉA, V., ROQUE-ROSELL, J., TORCHY, L., BINDER, D., SCIAU, P., PELEGRIN, J.,
REGERT, M., COUSTURE, M.-P., ROUCAU, C., 2012, Craft specialization and exchanges during the southern Chassey culture: an integrated archaeological and material sciences approach, en M. BORRELL, F. BORRELL, J. BOSCH, X. CLOP, M. MOLIST (dir.), Exchange of raw materials, products and ideas in the Western Mediterranean VIIIII millennium BC: Colloque international Networks in the Neolithic, Barcelone (Espagne), 2-4 Fév. 2011, Rubricatum 5, 119-129.

LEROI-GOURHAN A., 1964, Le Geste et la Parole 1, Technique et langage, París, Albin Michel.

LEROI-GOURHAN A., 1983, Le Fil du temps : ethnologie et préhistoire, 1935-1970, París, Fayard.

MAGET, M., 1953, Guide d'étude directe des comportements culturels, Paris, CNRS, Resolución de problemas, Investigación Bibliotecológica, vol. 26, n. ${ }^{\circ}$ 56, México.

MALLET, N., 1992, Le Grand-Pressigny: ses relations avec la civilisation Saône-Rhône, Supplément au Bulletin de la Société des Amis du Musée de Préhistoire du Grand-Pressigny 1.

MALLET, N., PELEGRIN, J., REDURONBALLINGER, M., 1994, Sur deux dépôts de lames pressigniennes: Moigny et Boutigny (Essonne), Bulletin des amis du musée de préhistoire du GrandPressigny 45, 25-37.

MANGADO, X., 2006, El aprovisionamiento en materias primas líticas: hacia una caracterización paleocultural de los comportamientos paleoeconómicos, Trabajos de Prehistoria 63, 79-91.

MANOLAKAKIS, L., 1994, La production des outils en silex dans les sociétés hiérarchisées de l'Enéolithique en Bulgarie : évolution, traditions culturelles et organisation sociale, Université Paris I-Sorbonne (tesis de doctorado).

MANOLAKAKIS, L., 2005, Les industries lithiques énéolithiques de Bulgarie, Internationale Archäologie, Band 88, Verlag Marie Leidorf Gmbh, Rahden/Westf., 314.

MARÍN DE ESPINOSA, J. A., GUTIÉRREZ, C., MARTÍN-LERMA, I., 2011, Análisis tecnológico del conjunto laminar de Cabezos Viejos (Archena, Murcia, España): una aproximación experimental, 
en A. MORGADO, J. BAENA PREYSLER y

D. GARCÍA GONZÁLEZ (eds.), La investigación experimental aplicada a la Arqueología, Universidad de Granada, Granada, 171-178.

MARÍN DE ESPINOSA, J. A., MARTÍN-LERMA, I., TARRIÑO, A., 2013, Estudio tecnológico del material lítico tallado procedente del yacimiento calcolítico "Casas del Cejo» (Lorca, Murcia), en A. PALOMO, R. PIQUÉ y X. TERRADAS (eds.), Experimentación en arqueología. Estudio y difusión del pasado, Sèrie Monogràfica del MAC 25.1, Girona, Museu d'Arqueologia de Catalunya, 247-256.

MARTÍNEZ, G., 1997, Late Prehistory Blade production in Andalusia (Spain), en A. RAMOS y M. A. BUSTILLO (eds.), Silicius Rocks and Culture, Universidad de Granada, Granada, 427-436.

MARTÍNEZ, G., MORGADO, A., 2005, Los contextos de elaboración de láminas prismáticas de sílex en Andalucía oriental durante el Neolítico reciente. Aspectos técnicos, modelos de trabajo y estructuración social, III Congreso del Neolítico en la Península Ibérica, Santander, 2003, Universidad de Cantabria, Santander, 359-368.

MELOGNO, P., 2012, La cientificidad de las ciencias de la información: fundamentación filosófica o resolución de problemas, Investigación Bibliotecológica, vol. 26, n. ${ }^{\circ}$ 56, México.

MERINO, J. M., 1969, Tipología lítica, Munibe XXI $(1,2,3), 1-325$.

MILLET-RICHARD, L. A., 1997, Habitats et ateliers de taille au Néolithique final dans la région du GrandPressigny (Indre-et-Loire). Technologie lithique, 2 vol., Université de Paris I Phanthéon-Sorbonne, París (tesis de nuevo doctorado).

NAMI, H. G., 2010, Experiments and Interpretation of Traditional Technologies: Essays in Honor of Errett Callahan, Ediciones de Arqueología Contemporánea, Buenos Aires, 91-168.

NAMI, H. G., 2011, Reflexiones epistemológicas sobre arqueología y tecnología lítica experimental, en A. MORGADO, J. BAENA y D. GARCÍA (eds.), La investigación experimental aplicada a la arqueología, Ronda, Universidad de Granada, 37-43.
NEWCOMER, M. H., 1975, Punch technique and upper paleolithic blades, en Lithic Technology: Making and Using Stone Tools, E. SWANSON (ed.), $9^{\text {th }}$ International Congress of Anthropological and Ethnological Sciences, Chicago, Mouton Publishers, La Haya, París, 97-105.

NILSSON, S., 1868, The primitive inhabitants of Scandinavia, Longmans, Green \& Co., Londres.

OLAUSSON, D., 1983, Experiments to Investigate the Effects of Heat Treatment on Use-wear on Flint Tools, Proceedings of the Prehistoric Society 49, $1-13$.

PELEGRIN, J., 1984a, Approche Technologique Expérimentale de la mise en forme de nucléus pour le débitage systématique par pression, en Préhistoire de la Pierre Taillée: 2 Économie du Débitage Laminaire, Cercle de Recherches et d'Études Préhistoriques, París, 93-103

PELEGRIN, J., 1984b, Débitage par Pression sur Silex : Nouvelles Expérimentations, en Préhistoire de la Pierre Taillée : 2 Économie du Débitage Laminaire, Cercle de Recherches et d'Études Préhistoriques, París, 117-127.

PELEGRIN, J., 1984c, Systèmes Expérimentaux d'inmobilisation du Nucléus pour le débitage par pression, en Préhistoire de la Pierre Taillée : 2 Économie du Débitage Laminaire, Cercle de Recherches et d'Études Préhistoriques, París, 105-116.

PELEGRIN, J., 1988, Débitage experimental par pression : du plus petit au plus grand, en J. TIXIER (dir.), Technologie Préhistorique, Notes et Monographies Techniques 25, 35-53.

PELEGRIN, J., 1991, Sur une Recherche Technique Expérimentale des Techniques de Débitage Laminaire, en Archéologie expérimental, tome 2, La Terre: l'os et la pierre, la maison et les champs, actes du Colloque International Expérimentation en archéologie: Bilan et perspectives, l'Archéodrome de Beaune, 6-9 avril 1988, Collection Ar, París, Éditions Errance, 118-128.

PELEGRIN, J., 1995, Technologie lithique : le Châtelperronien de Roc de Combe (Lot) et de la Côte (Dordogne), Cahiers du Quaternaire 20, Éditorial CNRS. 
PELEGRIN, J., 1997, Nouvelles observations sur le dépôt de lames de La Creusette (Barrou, Indreet-Loire), Bulletin de la Société des Amis du GrandPressigny 48, 19-34.

PELEGRIN J., 2000, Les techniques de débitage laminaire au Tardiglaciaire: critères de diagnose et quelques réflexions, en B. VALENTIN, P. BODU, y M. CHRISTENSEN (dir.), L'Europe centrale et septentrionale au Tardiglaciaire: confrontation des modèles régionaux de peuplement. Actes de la Table ronde internationale de Nemours, 14-16 mai 1997, APRAIF, Mémoires du Musée de Préhistoire d'Île de France 7, 73-85.

PELEGRIN, J., 2002, La production des grandes lames de silex du Grand Pressigny, en Matériaux, productions, circulations du Néolithique à l'Àge du Bronze, Séminaire du Collège de France, Éditions Errance, París, 131-148.

PELEGRIN, J., 2003, Blade-Making Techniques from the Old World: Insights and applications to mesoamerican obsidian lithic technology, en K. G. HIRTH (ed.), Mesoamerican Lithic Tecnhnology. Experimentation and Interpretation, Salt Lake City, 55-71.

PELEGRIN, J., 2006, Long blade technology in the Old World: an experimental approach and some archaeological results, en J. APEL y K. KNUTSSON (eds.), Skilled production and Social Reproduction. Aspects on Traditional Stone-tool Technology, Upsalla University Press, 37-68.

PELEGRIN J., 2012, New Experimental Observations for the Characterization of Pressure Blade Production Techniques, en P.M. DESROSIERS (ed.), The Emergence of Pressure Blade Making, Springer, Nueva York, 465-500.

PELEGRIN, J., RICHE, C., 1999, Un réexamen de la série de Bouvante (Drôme): matières premières lithiques et composantes technologiques, in circulations et identités culturelles alpines à la fin de la préhistoire, en

A. BEECHING (ed.), Valence, Centre d'Archéologie préhistorique, 183-196.

PELEGRIN, J., MORGADO, A., 2008, Grandes láminas, hojas y hojitas. Experimentación de la tecnología laminar de la Edad del Cobre del sur de la Península ibérica (IV-III mil. Cal. B.C.), en
A. MORGADO, J. BAENA y D. GARCÍA, II Congreso Internacional de Arqueología Experimental, Abstracts, Ronda, 26-28 noviembre 2008, 39-40.

PELEGRIN, J., IHUEL, E., 2005, Les 306 nucléus de la ruine de la Claisière (Abilly, Indre-et-Loire), Bulletin des Amis du Musée du Grand-Pressigny 56, 45-65.

PELEGRIN, J., MORGADO, A., 2007, Primeras experimentaciones sobre la producción laminar del Neolítico Reciente-Edad del Cobre del sur de la Península Ibérica, en M. L. RAMOS, GONZÁLEZ, J. E y J. BAENA, (eds.), Arqueología Experimental en la Península Ibérica: investigación, didáctica y patrimonio, Santander, Asociación Española de Arqueología Experimental, 131-139.

PELEGRIN, J., INIZAN, M. L., 2013, Soft hammerstone percussion use in bidirectional blade-tool production at Acila 36 and in bifacial knapping at Shagra (Qatar), Arabian Archaeology and Epigraphy 24, Issue 1, 79-86.

PERLÈS, C., 1987, Les industries lithiques taillées de Franchthi (Argolide, Grèce). Tome I: Présentation générale et industries paléolithiques, Excavations at Franchthi Cave, fasc. 3, Indiana University Press, Bloomington, Indianapolis.

PERLÈS, C., 1990, Les industries lithiques taillées de Franchthi (Argolide, Grèce). Tome II: Les industries du Mésolithique et du Néolithique initial. Excavations at Franchthi Cave, fasc. 5, Indiana University Press, Bloomington, Indianapolis.

PERLÈS, C., 2004, Les industries lithiques taillées de Franchthi (Argolide, Grèce), Tome III: Du Néolithique ancien au Néolithique final, Excavations at Franchthi Cave, fasc. 13, Indiana University Press, Bloomington, Indianapolis.

PERLÈS, C., 2012, Quand « diffusion » ne veut pas dire "interaction ", Actes del Congrés Internacional Xarxes al Neolitic, Rubricatum 5, 539-546.

RAHMANI, N., 2004, Nouvelle interprétation de la chronologie capsienne (Epipaléolithique du Maghreb), Bulletin de la Société Préhistorique Française 101(2), 345-360. 
REMICOURT, M., VAQUER, J., BORDREUIL. M., 2009, Production et diffusion au Chalcolithique des lames en silex du Ludien de Collorgues, Gard, Gallia Préhistoire 51, 213-244.

RICHE, C., 1998, Les ateliers de silex de Vassieux. Explotation des gîtes et diffusión des produits, Université de Paris X-Nanterre (tesis de $3^{\mathrm{r}}$ ciclo).

SÁNCHEZ, M., REY, M., RODRÍGUEZ, N., CASADO, A., MEDINA, B., MANGADO, X., 2014, The LITHICUB project: a virtual lithotheque of siliceous rocks at the University of Barcelona, Journal of Lithic Studies 1, 281-292.

SELLERS, G. E., 1886, Observations on Stonechiping, Smithsonian Report for 1885, 871-891.

SIRET, L., 1892, Nouvelle campagne de recherches archéologiques en Espagne, La fin de l'époque néolithique, L'Anthropologie III (4), 385-404.

TARRIÑO, A., 2001, El sílex en la Cuenca VascoCantábrica y Pirineo Navarro. Caracterización y su aprovechamiento en la Prehistoria, Universidad del País Vasco (tesis doctoral).

TERRADAS, X., PALOMO, A., CLOP, X., GIBAJA, J.F., 2005, Primeros resultados sobre el estudio de grandes hojas procedentes de contextos funerarios del noreste de la Península Ibérica, III Congreso del Neolítico en la Península Ibérica Santander 2003, 349-357.

TERRADAS, X., PALOMO, A., CLOP, X. 2009, Les grans fulles de sílex. Europa al final de la Prehistòria, Actes, Monografies 13, Museu d'Arqueologia de Catalunya, Barcelona, 35-45.

TEXIER, P.J., 1984, Un débitage expérimental de silex par pression pectorale à la béquille, Bulletin de la Société Préhistorique Française 8-1, 25-27.

TEXIER, P.J., MEIGNEN, L., 2011 , Soixante années de technologie lithique : étapes marquantes, apports et écueils, en F. DELPECH, J. JAUBERT (dir.), François Bordes et la Préhistoire: colloque international François Bordes : Colloque du CTHS, Bordeaux 22-24 avril 2009, París, Éditions du CTHS, Documents préhistoriques 29, 279-289.

TIXIER, J., 1963, Tipologie de l'Epipaléolithique de Maghreb, AMG, Mémorie du CRAPE, Alger II, París.
TIXIER, J., 1967, Procédés d'analyse et questions de terminologie concernant l'étude des ensembles industriels du paléolithique récent et de l'épipaléolithique dans l'afrique du nord-ouest, en W. W. BISHOP y J. DESMOND CLARK, (ed.) Background to evolution in Africa, University of Chicago Press, Chicago, 771-820.

TIXIER, J., 1972, Obtention de lames par débitage "sous le pied", Bulletin de la Société Préhistorique Française 69-5, 134-139.

TIXIER, J., 1974, Glossary for description of stone Tools, with special reference to the Epipaleolithic of the Maghreb, translated by M.H. Newcomer, Newsletter of lithic technology: special publication 1.

TIXIER, J., 1976, L'industrie lithique de l'Aïn Dokkara, Région de Tébessa, Lybica 24, 21-54.

TIXIER, J., 1978, Méthode pour l'étude des outillages lithiques : notice sur les travaux scientifiques de J. Tixier, Université de Paris X - Nanterre (doctorado de Estado).

TIXIER, J., (éd) 1984, Préhistoire de la pierre taillée 2; économie du debitage laminaire : technologie et expérimentation, París, CREP.

TIXIER, J., INIZAN, M.-L., ROCHE, H., 1980, Préhistorie de la Pierre Taillée 1 : Terminologie et Technologie, Cercle de Recherches et d'Études Préhistoriques, Valbonne Cedex.

TORQUEMADA, J. DE, 1615, Monarquía Indiana, edición de 1723.

VAQUER, J., 2007, Le rôle de la zone nord tyrrhénienne dans la difusion de l'obsidienne en Méditerranée nord-occidentale au Néolithique, en A. D'ANNA, J. CESARI, I. OGEL y J. VAQUER (eds.), Corse et Sardaigne préhistoriques relations et échanges dans le contexte méditerranéen, Actes du $128^{e}$ congrès national du CTHS de Bastia 2003, Éditions du CTHS, Documents préhistoriques 22, París, 99-119.

VAQUER, J., 2012, Réflexions sur échanges de biens matériels lithiques entre le midi de la France et le nord de la Péninsule Ibérique au Néolithique et au Chalcolithique, Congrés Internacional Xarxes al Neolític - Neolithic Networks Rubricatum, Revista del Museu de Gavà 5, 565-574. 
VAQUER, J., BRIOIS, F., 2006, La fin de l'Âge de Pierre en Europe du Sud : matériaux et productions lithiques taillées remarquables dans le Néolithique et le Chalcolithique du Sud de l'Europe, Actes de la table ronde de l'EHESS, Carcassonne, 5-6 sept. 2003, Éd. AEP, Tolosa.
VAQUER, J., REMICOURT, M., 2009, Productions et importations de grandes lames en silex au Néolithique et au Chalcolithique dans le midi de la France (4500-2400 AV. J-C.), en J. F. GIBAJA, X. TERRADAS, A. PALOMO, X. CLOP (eds.), Les grans fulles de sílex. Europa al final de la Prehistòria, Actes, Monografies 13, Museu d'Arqueologia de Catalunya, Barcelona, 35-45. 